\title{
Anectodal Report of Anomalous Circumflex Associated with Pathological Scintigraphy in a Patient with Atypical Chest Pain
}

\author{
Mariano $E^{*}$, Duro L, Sperandio M, Ros V, Viele A, Biscione C, Mango R and Romeo F
}

Department of Cardiology, University of Rome, Italy

\begin{abstract}
The incidence of Coronary Artery Anomalies (CAA) in routine Coronary Angiography(CAG) is between $0.6 \%$ and $1.5 \%$. Most anomalies are not of clinical significance, but some are related to angina, dyspnoea, syncope, acute myocardial infarction andsudden death. Diagnosis and adequate imaging of CAA are crucial for appropriate patient management, especially in patient candidates for Percutaneous Coronary Intervention (PCI) and cardiac surgery. This report describes the anomalous origin of the Left Circumflex Artery (LCX), arising by the right sinus of Valsalva and associated with the presence of hypoplasic Left Anterior Descending Coronary Artery (LAD) that correlates with myocardial scintigraphy findings of reduced uptake in the anterior and inferior wall of left ventricle.
\end{abstract}

Keywords: Coronary anomalies; Coronary angiography; Myocardial scintigraphy; MDCT

\section{Case Report}

A 75-year-old man affected by dyslipidemia, smoking hystory, hypertension and with family history of cardiovascular disease, was admitted to our hospital because of atypical chest pain episodes, whose symptoms onset goes back to 5 months ago. Physical examination revealed a heart rate 75 beats/min and a blood pressure of 130/75 $\mathrm{mmHg}$. Heart sounds were normal and there was a $2 / 6$ systolic ejection murmur at the apex. Laboratory results showed no abnormality and no raise of cardiac enzymes. Electrocardiography showed normal sinus rhythm with no ST-T changes. An echocardiogram revealed normal chamber size and left ventricular wall motion with mild mitral insufficiency. Holter electrocardiogram showed no arrhythmias. Actually, patient underwent stress/rest thallium-201 single-photon emission cardiac tomography (SPECT) that revealed, during exercise test, a defect in uptake of the radioisotope in the basal portion of the anterior wall and in the inferior side of the left ventricle, that completely regress during the rest phase (Figure 1). On the other hand, treadmill test showed ST depression in anterior precordial leads, in absence of symptoms. Finally, patient underwent CAG, in order to exclude suspicion for Coronary Artery Disease (CAD). Coronary angiography documented the absence of critical coronary narrowings, except for a mild plaque in the second segment of right coronary artery, associated with the presence of anomalous origin of the left circumflex artery, arising by the right sinus of Valsalva. Moreover, Left Descending Artery (LAD) was poorly represented in distal vascular bed and does not surround the apex of the left ventricle, while a well-developed intermediate branch (IR) supplied lateral myocardial wall (Figure 2A-C). In our opinion, the presence of CAA findings well explained radioisotope uptake defects assessed by myocardial scintigraphy. Considering the key role of the proximal course of the anomalous coronary artery in relation to the great vessel in prognostic stratification, we decided to perform a high resolution 64-slice Multi Detector Computed Tomography (MDCT) that clearly documented aberrant circumflex origin from right aortic sinus of Valsalva with a further retroaortic course of CX within the atrioventricular groove. The $\mathrm{Cx}$ was originating as a slit like ostia wih acute angulation from the right sinus. Anomalous LCX that represented the most frequent anatomic variant and is usually associated with a good outcome and a normal diameter of mean pulmonary artery (Figures $2 \mathrm{D}-\mathrm{F}$ and $3 \mathrm{~A}-\mathrm{C}$ ). Combined cardiac CT and SPECT were thus crucial techniques in determining mechanisms and proper therapy. The patient was treated with metoprolol, $200 \mathrm{mg}$ was given once daily with rapid improvement of angina symptoms. Patients six-months follow-up was event-free.

\section{Discussion}

Ectopic origin of the Cx artery from the right sinus of Valsalva or the right coronary artery was first described by Antopol and Kugel in 1933 with prevalence of $0.4-0.8 \%$ in angiographic series [1]. Coronary artery anomalies have been identified in 0.6 to $1.5 \%$ of coronary angiograms [2-3]. Anomalous origin of the circumflex artery from the right sinus of Valsalva is the common artery anomaly which is thought to be of no clinical significance per se. However, the recognition and adequate visualization of the anomaly is essential for proper patient management.

Definitive diagnosis of coronary anomalies is generally made by coronary angiography [4]. Although this anomaly is usually benign and asymptomatic it was reported that it may cause ischemia, sudden death and myocardial infarction. Failure to recognize the anomaly can be hazardous to patient management. This report describes the anomalous origin of the LCX [5], arising by the right sinus of Valsalva associated with the presence of small LAD, clearly correlating with SPECT findings. Coronary anomalies represent a rare clinical condition that may benefit from the integration of most advanced diagnostic techniques for appropriate clinical management and adequate selection of patients features suitable for PCI or cardiac surgery. Even if noninvasive investigations methods, such us myocardial scintigraphy, may recognize defects in radioisotope uptake, guiding selection of patients with highest suspicion for coronary artery disease, only invasive assessment by CAG settles as the gold standand technique for confirming diagnosis. Recently, these anomalies have been recognized using noinvasive or minimally invasive techniques like transthoracic and transesophageal echocardiography [6]. Furthermore, the variable clinical presentation and prognosis of coronary anomalies crucially

*Corresponding author: Enrica Mariano, Department of Cardiology, University of Rome, Italy, Tel: +39 06 49911; E-mail: enrica mariano@hotmail.com

Received May 13, 2015; Accepted July 20, 2015; Published July 27, 2015

Citation: Mariano E, Duro L, Sperandio M, Ros V, Viele A, et al. (2015) Anectoda Report of Anomalous Circumflex Associated with Pathological Scintigraphy in a Patient with Atypical Chest Pain. J Clin Case Rep 5: 560. doi:10.4172/21657920.1000560

Copyright: ( $) 2015$ Mariano E, et al. This is an open-access article distributed under the terms of the Creative Commons Attribution License, which permits unrestricted use, distribution, and reproduction in any medium, provided the original author and source are credited. 
Citation: Mariano E, Duro L, Sperandio M, Ros V, Viele A, et al. (2015) Anectodal Report of Anomalous Circumflex Associated with Pathological Scintigraphy in a Patient with Atypical Chest Pain. J Clin Case Rep 5: 560. doi:10.4172/2165-7920.1000560

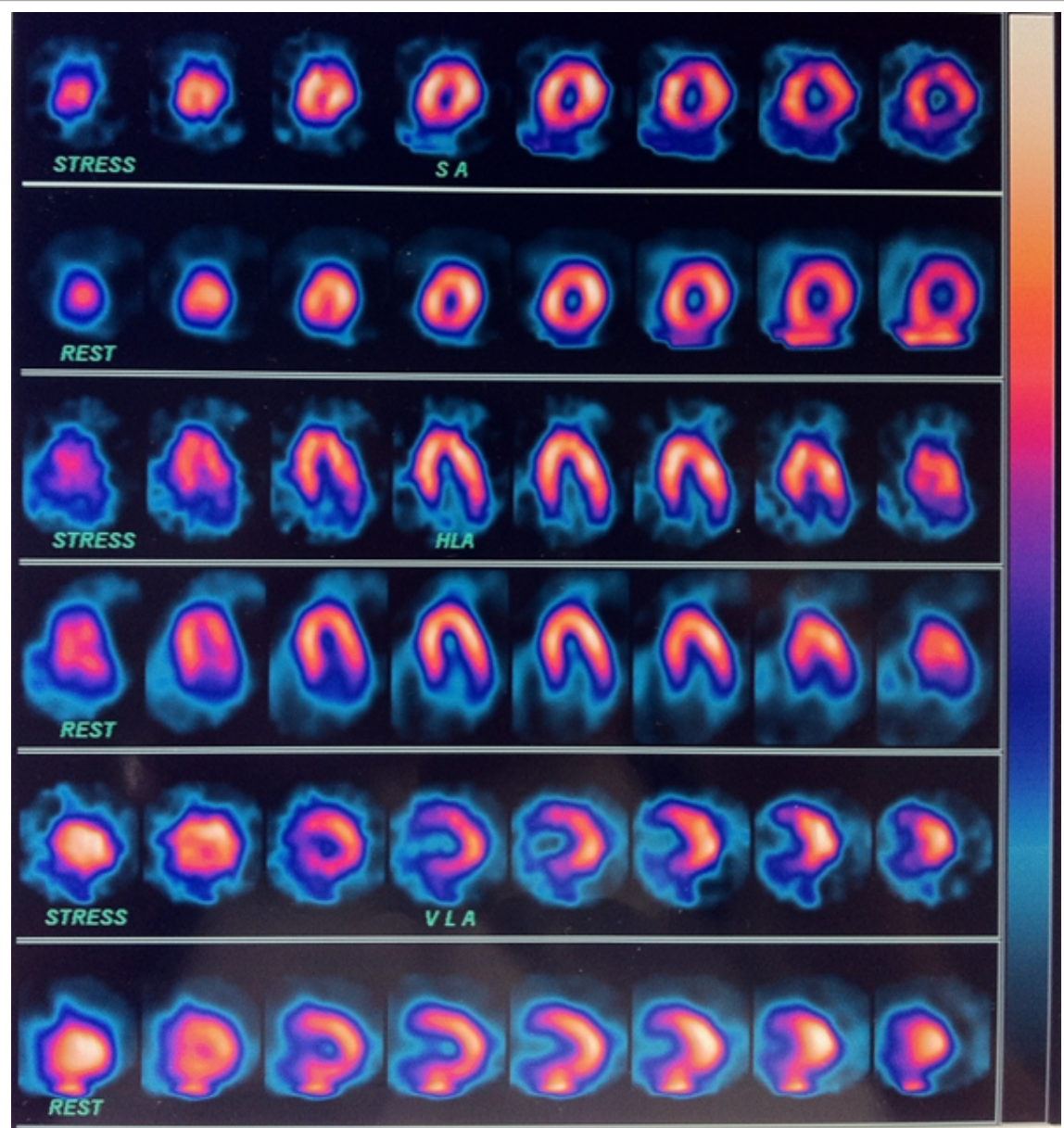

Figure 1: Stress/rest thallium-201 single-photon emission cardiac tomography (SPECT) showing reverisble perfusion defects in the basal portion of the anterior wall and in the inferior side of the left ventricle, that completely regress during the rest phase.
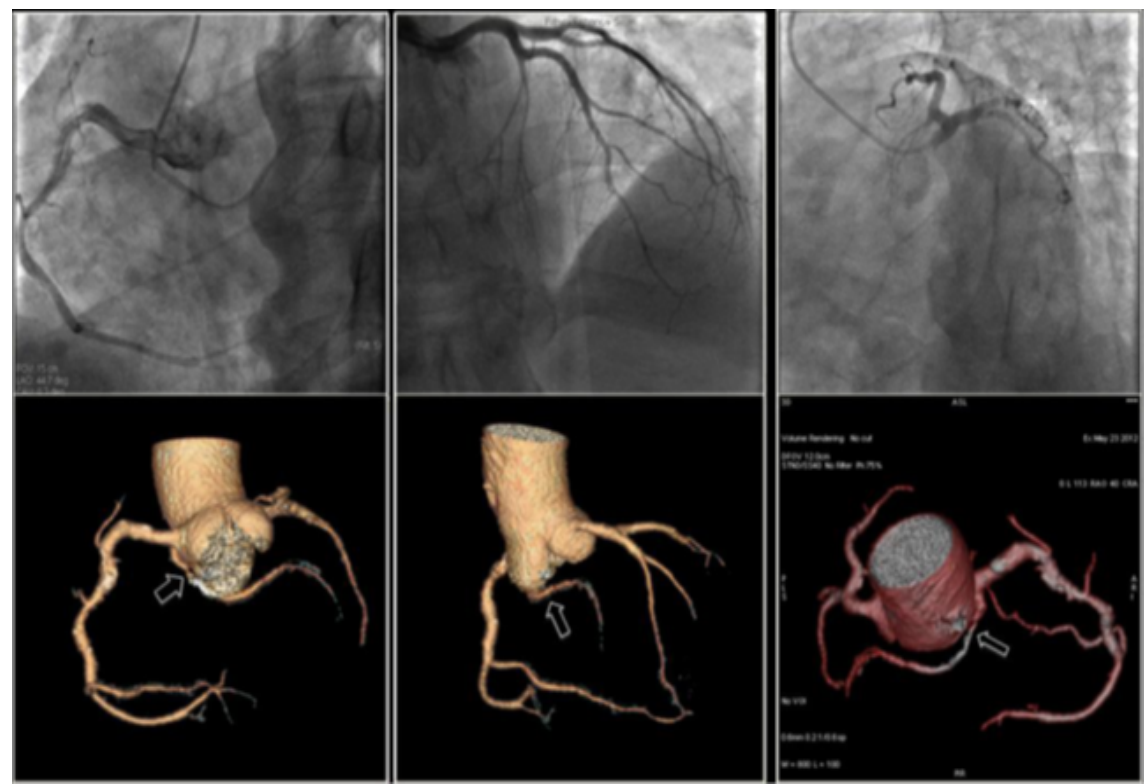

Figure 2: (A-C-Upper row) Coronary angiography documented the absence of critical coronary narrowings associated with the presence of anomalous origin of the left circumflex artery, arising by the right sinus of Valsalva. Left discending artery (LAD) was poorly represented in distal vascular bed and does not surround the apex of the left ventricle, while a well developed intermediate branch (IR) supplied lateral myocardial wall. (D-F-Lower row) Volume Reconstruction (VR) of the coronary tree. Those reconstructions demontraste the integrity of coronary vessels and the abnormal origin of Circumflex artery with retobulbar course. 
Citation: Mariano E, Duro L, Sperandio M, Ros V, Viele A, et al. (2015) Anectodal Report of Anomalous Circumflex Associated with Pathological Scintigraphy in a Patient with Atypical Chest Pain. J Clin Case Rep 5: 560. doi:10.4172/2165-7920.1000560
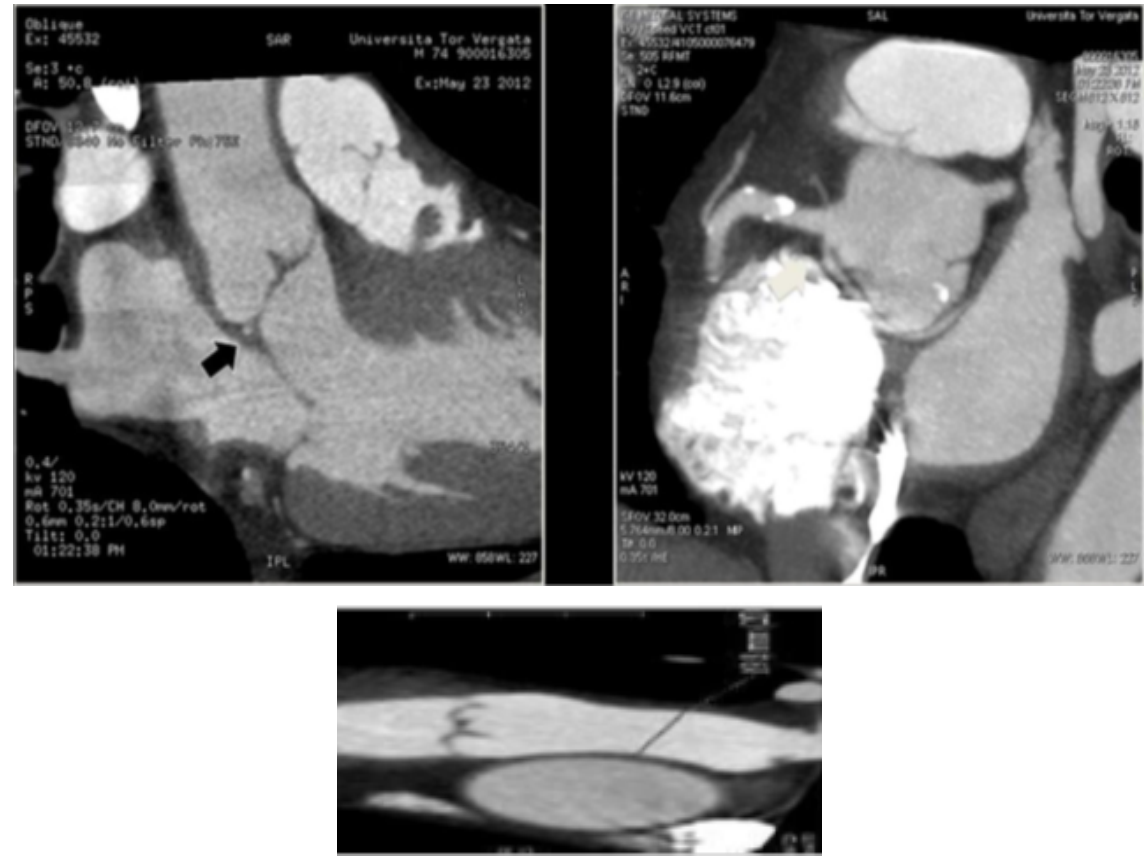

Figure 3: (a) MPR sagittal reconstruction shows the retrobulbar course of circumflex artery (white arrow). (b) Axial MIP reconstruction that shows the regular origin of main trunk and the origin of right coronary artery (RCA). Simultaneously the reconstruction demonstrates the abnormal origin of Circumflex artery from the first part of RCA (black arrow), with a retrobulbar aortic course of anomalous circumflex. (c) Contrast-enhanced computed tomographic axial immage of the main pulmonary artery measured transversely at the level of Pulmonary artery bifurcation shows normal diameter of the vessel $(<29 \mathrm{~mm})$. Note the additional feature of the absence of Pulmonary Hypertension: the ratio of Pulmonary artery to ascending aorta diameter does not exceeds 1.

depends on the proximal course [7] of the anomalous coronary artery in relation to the great vessels that can be accurately clarified by MDCT [8]. In conclusion, conventional CAG still represents the gold standard procedure for posing diagnosis of coronary artery anomalies while the integration with coronary MDCT is fundamental for defining anatomic variants.

\section{References}

1. Antopol W, Kugel MA (1933) Anomalous origin of the left circumflex artery. Am Heart J 8: 802-6.

2. Yamanaka O, Hobbs RE (1990) Coronary artery anomalies in 126,595 patients undergoing coronary arteriography. Cathet Cardiovascular Diagn 21: 28-40.

3. Aydinlar A, Ciçek D, Sentürk T, Gemici K, Serdar OA, et al. (2005) Primary congenital anomalies of the coronary arteries: a coronary arteriographic study in Western Turchei. Int Heart J 46: 97-103.

4. Konstantinos GG, P. Letsas, Loukas K. Pappas, loannis A, et al. (2007) Anomalous origin of the entire coronary system with separate ostia within the right sinus of valsalva: a rare congenital anomaly and a review of the literature. Cardiology 107: 209-212.

5. Young-Soo Lee (2006) Anomalous origin of the left circumflex coronary artery from the right sinus of Valsalva identified by imaging with multidetector computed tomography. Korean Circ J 36: 823-5.
6. Hale Y, Baris G, Sinan S, Osman B (2014) A case of anomalous origin of circumflex artery from right sinus of valsalva recognized by three-dimensionale transesophageal echocardiography and coronary computed tomography angiography. Heart views 15: 57-59.

7. Yuksel S, Meric M, Soylu K (2013) The primary anomalies of coronary artery origin and course: a coronary angiographic analysis of 16,573 patients. Exp Clin Cardiol 18: 121-1233.

8. Sato $\mathrm{Y}$, Inoue $\mathrm{F}$, Kunimasa $\mathrm{T}$ (2005) Diagnosis of anomalous origin of the right coronary artery using multislice computed tomography: evaluation of possible causes of myocardial ischemia. Heart Vessels 20: 298-300. 